\title{
Sagittal balance of the spine: consequences for the treatment of the degenerative spine
}

\author{
Max Aebi
}

Received: 11 July 2011/Published online: 5 August 2011

(C) Springer-Verlag 2011

I would like to thank Jean-Charles Le Huec and Pierre Roussouly for making the enormous effort to put together a comprehensive body of work, which reflects the state of the art about the today's knowledge and understanding of the "Sagittal Balance" of the spine.

This supplement is another documentation of the outstanding thought school of the French orthopaedic surgery, which has provided us with fundamental knowledge and innovation in orthopaedic surgery in general, but in spinal surgery and science in particular. We should not forget that century knowledge like pedicle fixation has its origin mainly in the French orthopaedic surgery in the second half of the 20th century, long before the American Academy named their annual congress in 1987 the "year of the pedicle".

That the sagittal balance is one of the most mysterious facts in the evolution of man we know since long, but it has never really been approached in the rigorous scientific way as it is outlined in this supplement. The human posture has inspired philosophers, poets and physicians already in ancient time and some have concluded from the individual physical posture of man in the sagittal plane to his "inner" attitude: proudness, timidity, cowardice, etc.
For us as physicians, surgeons and researchers it will be crucial to understand whether the physical posture implies and causes certain pathologies of the spine, in particular degenerative disease. The next question then will be whether such pathology can be prevented or treated by adapting the sagittal alignment and the spinal-pelvic relationship.

Only when we may end up in answering these questions, the measuring of angles and the combination of different anatomical axes will make sense. Otherwise, it would remain l'art pour l'art. This supplement should give us knowledge about these questions, initiate new questions and provoke answers-the rightly asked question is very often more important and powerful than the answer-in order to understand whether this new "science" is of clinical help.

I wish our readers to find all this in this supplement.

M. Aebi

Editor-in-Chief

Conflict of interest None. 